Check for updates

Cite this: RSC Adv., 2019, 9, 2822

\title{
3D printed fittings and fluidic modules for customizable droplet generators $\uparrow$
}

\author{
Sindhu Vijayan ${ }^{\mathrm{ab}}$ and Michinao Hashimoto (D) *ab
}

We developed a rapid and simple method to fabricate microfluidic non-planar axisymmetric droplet generators using 3D printed fittings and commercially available components. 3D printing allows facile fabrication of microchannels albeit with limitations in the repeatability at low resolutions. In this work, we used 3D printed fitting to arrange the flow in the axisymmetric configuration, while the commercially available needles formed a flow-focusing nozzle as small as $60 \mu \mathrm{m}$ in diameter. We assembled 3D printed fitting, needle, and soft tubes as different modules to make a single droplet generator. The design of our device allowed for reconfiguration of the modules after fabrication to achieve customized generation of droplets. We produced droplets of varying diameters by switching the standard needles and the minimum diameter of droplet obtained was $332 \pm 10 \mu \mathrm{m}$ for $34 \mathrm{G}$ (ID $=60 \mu \mathrm{m})$. Our method allowed for generating complex emulsions (i.e. double emulsions and compartmented emulsions) by adding 3D printed sub-units with the fluidic connections. Our approach offered characteristics complementary to existing methods to fabricate flow-focusing generators. The standardized needles serving as a module offered well-defined dimensions of the channels not attainable in desktop 3D printers, while the 3D printed components, in turn, offered a facile route to reconfigure and extend the flow pattern in the device. Fabrication can be completed in a plug-and-play manner. Overall, the technology we developed here will provide a standard approachable route to generate customized microfluidic emulsions for specific applications in chemical and biological sciences.

Received 19th October 2018

Accepted 9th January 2019

DOI: $10.1039 / c 8 r a 08686 a$

rsc.li/rsc-advances

\section{Introduction}

This paper describes a rapid and simple method to fabricate microfluidic non-planar axisymmetric droplet generators using 3D printed fittings and commercially available needles and tubes. Droplets can be manipulated using microfluidic devices that allow one phase to be dispersed in another immiscible phase. ${ }^{1}$ Droplet microfluidics has evolved as a multidisciplinary platform with a broad spectrum of applications in the fields of analytical chemistry, ${ }^{2,3}$ materials synthesis, ${ }^{4-8}$ drug delivery, ${ }^{9-11}$ biological assays, ${ }^{12-14}$ imaging $^{15,16}$ and food chemistry. ${ }^{17,18}$ Microfluidic droplet generators have been widely used to produce highly controlled and monodisperse droplets with various degree of complexity.

Fabrication of such droplet generators has been demonstrated primarily in three different routes: (1) soft lithography and replica molding ${ }^{19-21}$ (2) 3D printing ${ }^{22-27}$ and (3) manual assembly of fluidic units. ${ }^{28-34}$ Soft lithography employs

${ }^{a}$ Pillar of Engineering Product Development, Singapore University of Technology and Design, 8 Somapah Road, Singapore 487372, Singapore. E-mail: hashimoto@sutd. edu.sg

${ }^{b}$ Digital Manufacturing and Design (DManD) Centre, Singapore University of Technology and Design, 8 Somapah Road, Singapore 487372, Singapore

$\dagger$ Electronic supplementary information (ESI) available. See DOI: 10.1039/c8ra08686a photolithography followed by replica molding with polydimethylsiloxane (PDMS). Fabrication of microfluidic devices in cleanroom is relatively expensive and laborious. ${ }^{24}$ In addition, replica molding creates primarily planar devices where surface wettability and solvent compatibility need to be optimized to achieve the stable generation of droplets. To this end, additional modifications of the surface of channels are required to maintain preferred surface properties, solvent resistance, and wettability. ${ }^{35-38}$ Alternatively, the recent advance in 3D printing has allowed fabricating microfluidic devices designed in computer-aided design (CAD) files. ${ }^{22-27}$ In stereolithography and polymer jetting 3D printing, the evacuation of the uncured resin or the support materials to form narrow channels is often challenging. ${ }^{23-25,27}$ Multistep postprocessing to remove uncured resin ${ }^{23}$ or support material ${ }^{27}$ is often required. This limitation hampers the resolution, and complexity of the channels and orifices in the 3D printed droplet generators. Recently, the clogging of resin in fully 3D printed devices was addressed by printing the entire device in small modules that can be post-assembled. ${ }^{27,39}$ In another way, flow-focusing droplet generators can be fabricated by assembling subunits. For example, glass capillaries are arranged in the co-axial configuration to form a droplet generator..$^{28,31,32}$ The capillary devices made of glass have been successfully employed for the generation of single and multiple emulsions, 
while the alignment of glass capillaries poses the difficulty in fabrication and operation. ${ }^{25,26}$ Manual assemblies of the subunits such as tubes, needles, and adapters allowed creating low-cost microfluidic droplet generators suitable for generating single emulsions ${ }^{29,30,33}$ and double emulsions. ${ }^{40}$ While the use of commercially available parts as the subunits makes the fabrication simple, the fabricated devices are limited in their complexity of the configuration of the channel, which in turn limits the complexity of the emulsions that can be generated.

At present, there is a trade-off between the ease of fabricating droplet generators and the size and complexity of the emulsions that can be generated. We addressed this limitation by combining simple 3D printed modules and commercially available parts (such as needles and tubes) with a small dimension to fabricate axisymmetric droplet generators. The use of 3D printed modules offered integrating large microchannels (greater than $500 \mu \mathrm{m}$ ) with branches and other structures, and the use of commercially available needles and tubes offered a facile way to create fluidic connections with predetermined dimensions (as small as $60 \mu \mathrm{m}$ in the circular diameters). As we employed a modular approach, the device was assembled and modified in a plug-and-play manner. This capability allowed customizing the inner diameter (ID) of the flow-focusing nozzles (from $60 \mu \mathrm{m}(34 \mathrm{G})$ to $1.4 \mathrm{~mm}(14 \mathrm{G})$ ) merely by replacing the needles. The reconfiguration of the flow-focusing nozzles in serial and parallel arrangement permitted generating complex, higher-order emulsions. The simplicity of this method should enable controlled microfluidic generation of droplets accessible in various research fields.

\section{Experimental design}

The objective of this research is to develop a simple method to fabricate a non-planar axisymmetric droplet generator by assembling readily available modules to generate complex emulsions. The non-planar geometry of the flow-focusing device allows for the stable generation of droplets irrespective of the combination of fluids used as the outer and the inner phases. Specifically, we proposed to develop a method that is advantageous in the following three aspects: (1) ease of the fabrication, (2) resolution of the channel, and (3) customizability of the channel.

In order to ensure the ease of fabrication, we adopted a modular approach for fabrication. The different modules employed to form an axisymmetric droplet generator were 3D printed fittings and readily available low-cost materials such as needles and tubes. Rigid fittings fabricated by 3D printing was readily assembled with other modules, while the elasticity of the tube naturally provided conformal contact to avoid leakage of the fluids. Other than connecting different modules together, no additional procedure was involved to make the flow-focusing device. The 3D printed fitting was designed with large hollow spaces so that resin clogging should not occur, unlike fully 3D printed devices with embedded microchannels. ${ }^{23-25}$
Secondly, one of the current limitations of the 3D printed microchannels is the attainable resolutions. We overcome this limitation by integrating standardized needles into the channel system. Standardized needles were available with smaller inner diameters $(60 \mu \mathrm{m})$ than the printable channel widths of the $3 \mathrm{D}$ printed device. ${ }^{22-25}$ The use of standard needles with fixed apertures also ensured the repeatability of fabrication. The diameters of the needles can be selected from $60 \mu \mathrm{m}(34 \mathrm{G})$ to $1.4 \mathrm{~mm}(14 \mathrm{G})$.

Finally, it is difficult to alter the configuration of the device after fabrication in a completely $3 \mathrm{D}$ printed device ${ }^{22-25}$ as the channels are fixed and there is no modifiable component. In our approach, the modification of the assembled device was achieved for the geometry of the channels (with different 3D printed fittings) as well as the dimension of the nozzles (with different standardized needles). The use of 3D printed fittings (as compared to simple tubes as fittings) allows altering the type of droplets produced. It was feasible to add multiple devices and couple other customized 3D printed fittings (such as a Y-channel) for the generation of higher-order and complex emulsions, which would not be possible in the simple assembly of tubes and needles. ${ }^{29,30}$

\section{Results and discussion}

\section{Fabrication of axisymmetric droplet generator using 3D printed fittings}

We fabricated a non-planar axisymmetric droplet generator using the modules of $3 \mathrm{D}$ printed fitting and readily available low-cost components. The fabricated axisymmetric droplet generator was tested for its customizability to produce simple and complex emulsions. Initially, we checked the feasibility to design and fabricate the modular devices; a single droplet generator comprising a rigid $3 \mathrm{D}$ printed connector, a dispensing needle and soft tubes for connections are illustrated (Fig. 1a). The dimensions of the modules used for the fabrication of axisymmetric flow-focusing devices are given (Fig. S1†). The rigid connector was designed as a hollow cylinder with an inlet to permit the fluid to flow. The droplet generator was made by co-axially aligning the $3 \mathrm{D}$ printed connector and dispensing needle with fluidic connections for inlet and outlet (Fig. 1b). We used elastic soft tubes (made of silicone rubber) as an outlet to establish tight connections between the needle and connector. This arrangement permitted to prevent leakage of the fluid. We further extended our device to generate complex and higher-order emulsions. The flow-focusing nozzles were connected in series by arranging two flow-focusing units to produce multiple emulsions (i.e. emulsions encapsulated in other emulsions) (Fig. 1c). Alternatively, the flows can be arranged in parallel using a 3D printed branch (Y-channel) as a fitting. This fitting allowed to form a laminar flow of two parallel streams of fluids as a single dispersed phase for the droplet generation (Fig. 1d). Due to rigidness and large void space of the 3D printed fittings, we were able to clean the fittings easily with water and reassemble them for multiple usages. 
a)

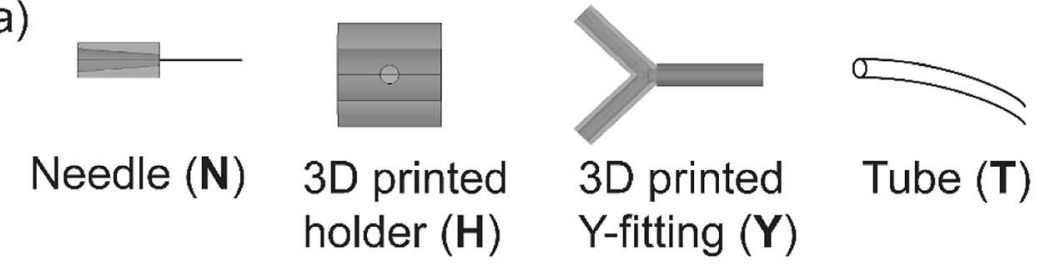

b) Single

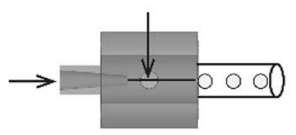

c) Serial
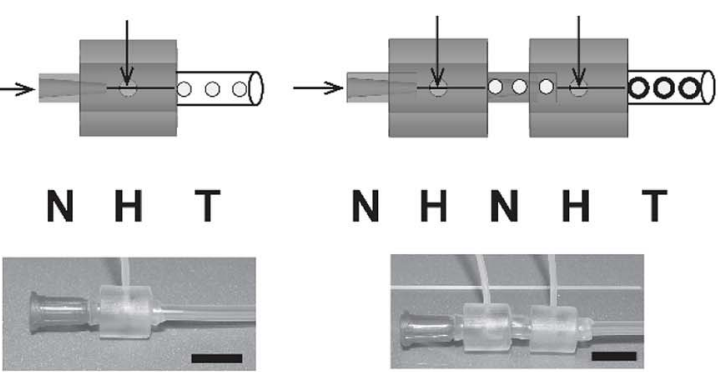
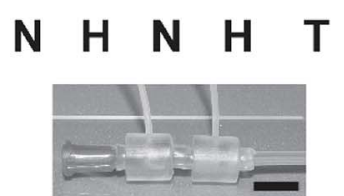

d) Parallel

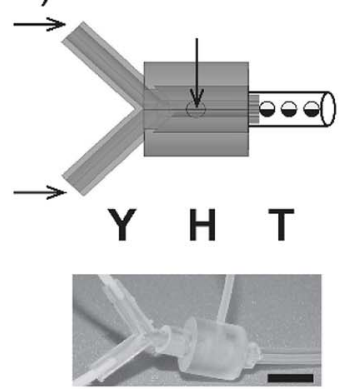

Fig. 1 (a) Modules used for the fabrication of microfluidic axisymmetric flow-focusing device: needle (N), 3D printed holder (H), 3D printed $Y$ fitting $(Y)$ and tube $(T)$. Schematics and optical images of (b) a single flow-focusing device formed by co-axial arrangement of the 3D printed connector and a needle, (c) serially connected, two flow-focusing devices to produce double emulsions, and (d) a flow-focusing device with a parallel laminar flow to produce compartmented particles. Scale bar $=10 \mathrm{~mm}$.

\section{Customization of flow-focusing nozzle}

We demonstrated the advantage of our approach to assemble and reconfigure the modules of the flow-focusing droplet generators. A single flow-focusing droplet generator was fabricated by assembling the modules such as rigid 3D printed connector, dispensing needle and soft tubes. The commercially available dispensing needles vary in their inner diameters (ID) from a few tens of micrometers to a few millimeters. We switched the needle assembled with the flow-focusing device and our design allowed for this reconfiguration without altering the geometry. By this easy reconfiguration, we generated droplets of varying diameters depending on the ID of the needle for the same flow conditions (i.e. type of fluids and flowrate). The single flow-focusing device was experimented with three different gauges of needles (25 G, $30 \mathrm{G}$ and $34 \mathrm{G}$ with corresponding IDs of $250 \mu \mathrm{m}, 160 \mu \mathrm{m}$, and $60 \mu \mathrm{m})$. Water-in-oil droplets $(\mathrm{W} / \mathrm{O})$ were formed using hexadecane containing Span $80(2 \% \mathrm{v} / \mathrm{v})$ as the continuous phase and aqueous solution containing blue dye $(1 \% \mathrm{v} / \mathrm{v})$ as the dispersed phase. The flowrate of the dispersing fluid $\left(Q_{\mathrm{d}}\right)$ was maintained at $10 \mu \mathrm{L} \mathrm{min}^{-1}$, while the continuous fluid $\left(Q_{c}\right)$ was varied between 100

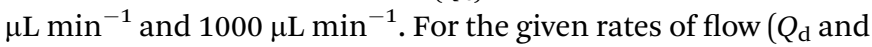
$Q_{\mathrm{c}}$ ), the diameter of droplets decreased with the decrease in the ID of needles (Fig. 2a). We studied the effect of $Q_{\mathrm{c}}$ on the diameter of the formed droplet for needles of three different size and the fixed $Q_{\mathrm{d}}$. The diameter of droplets decreased with the increase in $Q_{\mathrm{c}}$ (Fig. 2b). This observation is in accordance with previous studies carried out in other microfluidic flowfocusing devices fabricated by other methods. ${ }^{\mathbf{2 0 , 2 4}}$ The minimum diameter of droplet attained in our study was $332 \pm$ $10 \mu \mathrm{m}$ for $34 \mathrm{G}(\mathrm{ID}=60 \mu \mathrm{m})$ at the flowrates of $1000 \mu \mathrm{L} \mathrm{min} \mathrm{m}^{-1}$

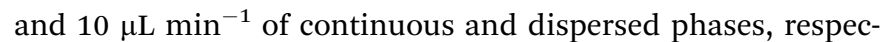
tively. The minimum diameter of droplets obtained in our device was smaller than those obtained using fully 3D printed devices with the orifices larger than the diameter of needle. ${ }^{23,25}$

Our method to fabricate axisymmetric flow-focusing droplet generator offered three major advantages over 3D printing of the entire microfluidic flow-focusing generators. Firstly, in our work, the ID of the standard needles used for the dispersing fluid was smaller than the width of channels obtained in a fully 3D printed non-planar device. ${ }^{23,25,27}$ Secondly, the large void volume of $3 \mathrm{D}$ printed connector used in our work prevented uncured resin from clogging the space; the clogging of resin was reported to be a problem in narrow channels embedded in fully 3D printed device. ${ }^{24,27}$ Thirdly, our flow-focusing device allowed for reconfiguration after fabrication by altering its modules which is not feasible in a fully printed device. In our approach, it was possible to change the diameter of droplets only by changing the needles with varying IDs. We demonstrated that the IDs of needles connected to the device could be changed from $60 \mu \mathrm{m}$ to $250 \mu \mathrm{m}$, which were smaller than the minimum width of the channel of fully $3 \mathrm{D}$ printed microchannels (reported as a few hundred micrometers). ${ }^{23,25}$

\section{Formation of double emulsions by serial connections}

Next, we demonstrated the customizability of the device using our method. We showed that the single flow-focusing device could be readily extended in series (back to back) to produce multiple emulsions. To demonstrate the proof-of-principle, we assembled two identical devices in series to produce double emulsions. Soft elastic tubes ensured a tight connection between the two devices. The needles of the two connected 
a)

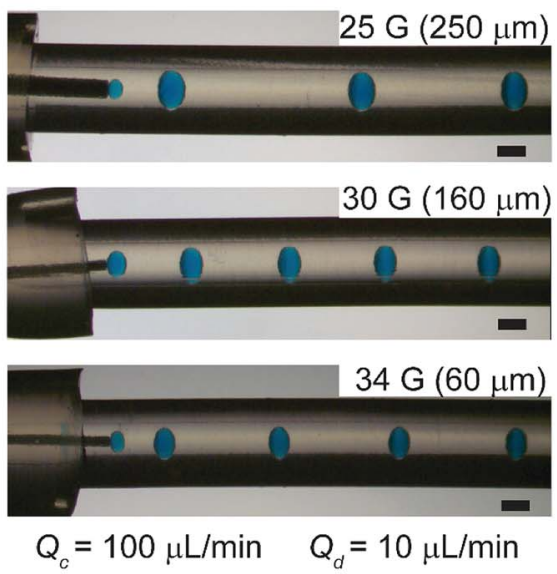

b)

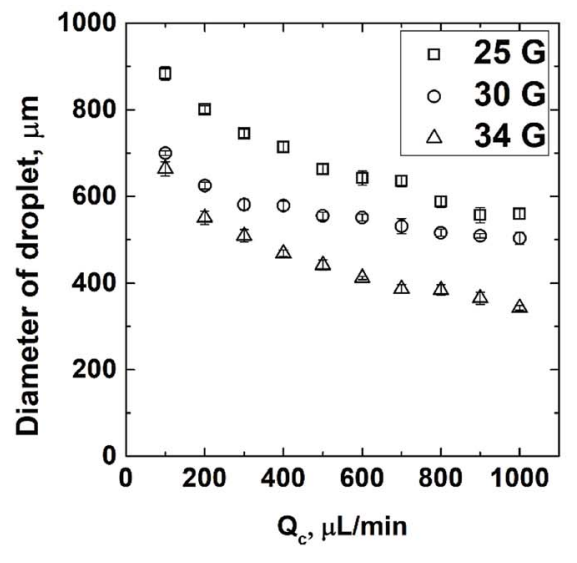

Fig. 2 (a) Optical micrographs showing the controlled generation of W/O emulsions using three different dispensing needles of varying IDs. (b) A plot showing the diameter of droplets obtained for three different dispensing needles against varying $Q_{c}$ at $Q_{d}=10 \mu \mathrm{L}$ min $^{-1}$. The dispersed phase was $1 \% \mathrm{v} / \mathrm{v}$ blue dye in water, and the continuous phase was $2 \% \mathrm{v} / \mathrm{v}$ Span 80 in hexadecane. Scale bar $=600 \mu \mathrm{m}$.

devices were aligned co-axially to produce water-in-oil-in-water $(\mathrm{W} / \mathrm{O} / \mathrm{W})$ double emulsions with varying numbers of inner droplets at different flow conditions (Fig. 3). In a preceding study, multiple needles and PDMS block were successfully employed to form double emulsions while the alignment was completed manually in an uncured PDMS. ${ }^{40}$ In our work, the use of standard components and straightforward assembly guided by the 3D printed fitting and opening ensured rapid manual assembly of the devices with repeatability.

Stable generation of double emulsions by connecting the same type of devices in series confirmed the co-axial arrangement of the flows formed in the non-planar devices. In a planar 3D printed device, surface modification would be required to change the wettability of channels to produce multiple emulsions. ${ }^{26}$ An axisymmetric flow-focusing device permits generation of both oil-in-water $(\mathrm{O} / \mathrm{W})$ and water-in-oil $(\mathrm{W} / \mathrm{O})$ in the same device. Devices made by the alignment of the capillaries $^{31,32}$ and 3D printing ${ }^{26}$ were subjected to adequate surface treatment to produce double emulsions. Surface treatment was carried out in such devices to ensure that the outer fluid entering the collection tube preferentially flow along the surface while encompassing emulsions in an axisymmetric configuration. Our work successfully produced double emulsions by connecting two flow-focusing modules in series without any additional steps to modify the surface.

\section{Formation of bi-compartmented particles by parallel connection}

Finally, we demonstrated that the 3D printed fitting offered a specific arrangement of the flow to achieve the generation of complex emulsions. As a demonstration, we produced Janus particles by establishing co-laminar flow using a 3D printed fitting. Microfluidic devices used for producing monodisperse Janus particles were fabricated in different approaches including dry-etching, ${ }^{41}$ soft lithography ${ }^{42}$ and alignment of capillaries. ${ }^{43}$ To create a laminar flow consisting of two fluids in our device, we fabricated a fitting enclosing a branched Ychannel. The Y-channel was arranged coaxially with the connector where the continuous fluid was introduced. Two streams of the poly(ethylene glycol) diacrylate (PEGDA) solutions (colored in green and red) containing a photoinitiator formed a laminar flow that served as a single dispersed phase. a)

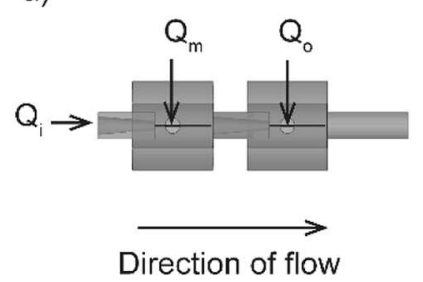

b)

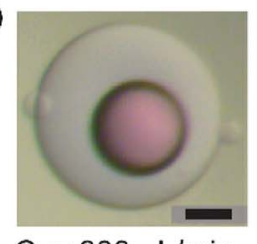

$Q_{o}=600 \mu \mathrm{L} / \mathrm{min}$

$Q_{m}=30 \mu \mathrm{L} / \mathrm{min}$

$Q_{i}^{m}=5 \mu \mathrm{L} / \mathrm{min}$

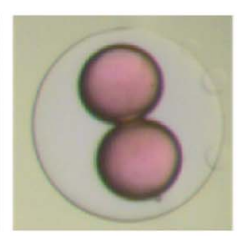

$Q_{0}=500 \mu \mathrm{L} / \mathrm{min}$ $Q_{m}=30 \mu \mathrm{L} / \mathrm{min}$ $Q_{i}=5 \mu \mathrm{L} / \mathrm{min}$

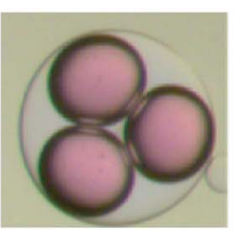

$Q_{0}=400 \mu \mathrm{L} / \mathrm{min}$

$Q_{m}=30 \mu \mathrm{L} / \mathrm{min}$

$Q_{i}^{m}=5 \mu \mathrm{L} / \mathrm{min}$
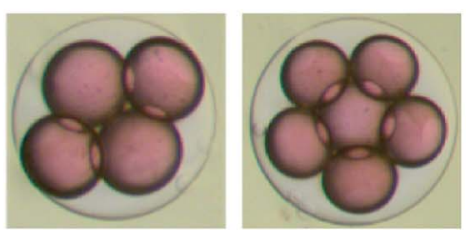

$Q_{0}=300 \mu \mathrm{L} / \mathrm{min} Q_{0}=200 \mu \mathrm{L} / \mathrm{min}$

$Q_{m}=30 \mu \mathrm{L} / \mathrm{min} \quad Q_{m}=30 \mu \mathrm{L} / \mathrm{min}$

$Q_{i}^{m}=5 \mu \mathrm{L} / \mathrm{min} \quad Q_{i}^{m}=5 \mu \mathrm{L} / \mathrm{min}$

Fig. 3 (a) A schematic illustration of serially connected flow-focusing devices for producing double emulsions. (b) Optical micrographs of produced W/O/W double emulsions with varying numbers of inner droplets obtained for a range of $Q_{0}$ with $Q_{m}=30 \mu \mathrm{L} \mathrm{min}^{-1}$ and $Q_{\mathrm{i}}=5$

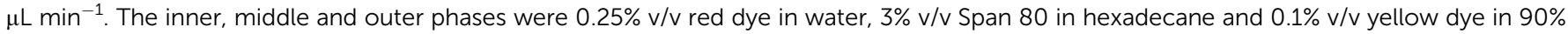
glycerol in water solution. Scale bar $=600 \mu \mathrm{m}$. 
a)

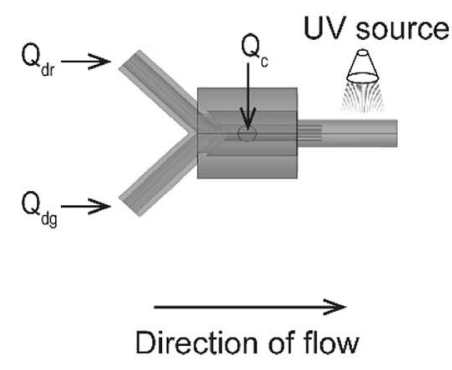

b)

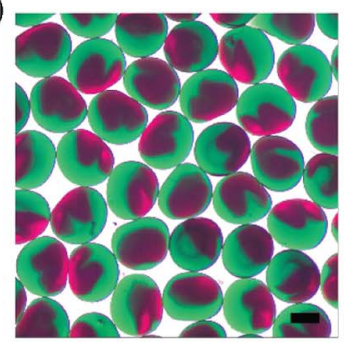

$Q_{c}=300 \mu \mathrm{L} / \mathrm{min}$

$Q_{d g}=35 \mu \mathrm{L} / \mathrm{min}$

$Q_{d r}=10 \mu \mathrm{L} / \mathrm{min}$

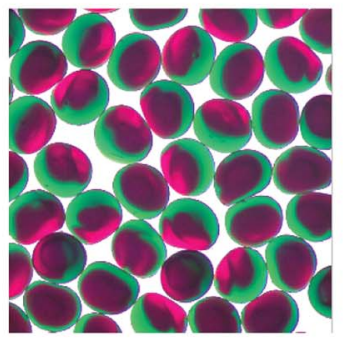

$Q_{c}=300 \mu \mathrm{L} / \mathrm{min}$

$Q_{d g}=25 \mu \mathrm{L} / \mathrm{min}$

$Q_{d r}=10 \mu \mathrm{L} / \mathrm{min}$

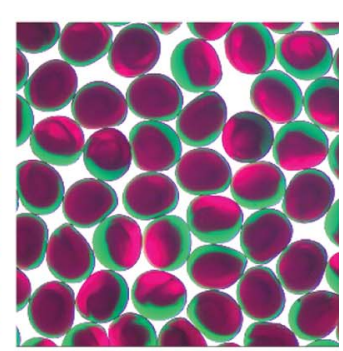

$Q_{c}=300 \mu \mathrm{L} / \mathrm{min}$

$Q_{d g}=15 \mu \mathrm{L} / \mathrm{min}$

$Q_{d r}=10 \mu \mathrm{L} / \mathrm{min}$

Fig. 4 (a) A schematic illustration of flow-focusing device connected with Y-channel as an inlet of the dispersed phase for producing bicompartmented Janus particles. (b) Optical micrographs of produced compartmented particles at three conditions of flows. Scale bar $=300 \mu \mathrm{m}$.

The dispersed phase comprising of two-colored fluids pinched off at the nozzle, and the compartmented droplets were immediately photopolymerized by irradiation of UV light. The flowrate of the red stream $\left(Q_{\mathrm{dr}}\right)$ was maintained at $10 \mu \mathrm{L} \mathrm{min}{ }^{-1}$ whereas three flowrates used for the green stream $\left(Q_{\mathrm{dg}}\right)$ were 35 , 25 and $15 \mu \mathrm{L} \mathrm{min}^{-1}$. We obtained bi-compartmented particles with different proportions of the red and green compartments for the given three different conditions of flows (Fig. 4).

Janus particles produced in planar microfluidic devices consists of compartments in the two-dimensional plane. ${ }^{42}$ The 3D printed fitting (Y-channel) designed with two channels of inlets enabled generation of particles with two compartments in this experiment. The non-planar geometry of our device, in principle, would enable the formation of spherical compartmented particles in the three-dimensional arrangement. 3D printing helps to fabricate fittings with multiple inlets of uniform size and geometry to achieve desired flow patterns. Attaining uniform sized orifices with defined geometry would be challenging in a device with manually cut capillaries. Using an appropriate 3D printed fitting, we can generate multicompartmented particles having diverse properties for specific applications such as drug carriers ${ }^{44,45}$ and semiconducting beads. ${ }^{46}$

\section{Conclusions}

In this paper, we reported a simple modular approach to fabricate axisymmetric microfluidic flow-focusing devices for the generation of simple and complex emulsions. The fabrication of the device was achieved rapidly by assembling the 3D printed subunits and commercially available parts, while we achieved stable generation of complex emulsions (i.e. double emulsions and compartmented emulsions) in the same device. With the wide range of needles available with different aperture sizes, we showed that the device can be reconfigured to produce droplets of varying diameters. The design of the device facilitated the reconfiguration of the modules after the device was fabricated. We obtained the minimum diameter of the droplet as $332 \pm 10 \mu \mathrm{m}$ using the smallest needle with an ID of $60 \mu \mathrm{m}$. The aperture size of the needles used in our work was smaller than the width of the channels in a fully $3 \mathrm{D}$ printed device. ${ }^{25}$
The modules could be arranged to form multiple and complex emulsions; two flow-focusing generators placed in series allowed producing double emulsions, and a laminar flow consisting of parallel flows of two fluids was used to generate Janus particles. Our approach successfully balanced the ease of fabrication of microfluidic flow-focusing devices and the complexity of emulsions obtained. The fabricated devices can be easily cleaned with water and reused multiple times.

The method of fabrication we developed offers several characteristics that would complement existing methods to fabricate flow-focusing generators. We took an advantage of well-defined, small size of features that the standardized materials (i.e. needles and tubes) offered, which were not achievable in desktop 3D printers. 3D printed fitting added the route to design the configuration of the multiphase flows (i.e. concentric and co-laminar) to achieve generation of intended emulsions. As the fabrication can be done in a simple plug-andplay manner, we believe that the method we developed in this work benefits non-experts to perform generation of emulsions using microfluidic devices for various applications.

\section{Materials and methods}

\section{Design and fabrication of $3 D$ printed fittings}

3D fittings (connector and Y-channel) were designed using AutoCAD® 2016 (Autodesk, USA). The connector was in the shape of a hollow cylinder (length $=10 \mathrm{~mm}, \mathrm{OD}=10 \mathrm{~mm}$, and $\mathrm{ID}=5 \mathrm{~mm}$ ). The hollow connector was designed with a circular hole of inner diameter $1.8 \mathrm{~mm}$ at the side for the flow of continuous fluid. The Y-channel had two inlets opening and the outlet of $1.8 \mathrm{~mm}$ in outer diameter. Two polytetrafluoroethylene (PTFE) tubes (muse 2000, China) with ODs and IDs of (1.8 mm, $1 \mathrm{~mm})$ and $(1 \mathrm{~mm}, 0.7 \mathrm{~mm})$ were fitted concentrically to the outlet of Y-channel that delivered the co-laminar flow. The CAD files were exported in the standard triangulation language (STL) file format. STL files of the design were sent to Form $2^{\mathrm{TM}}$ (Formlabs, USA) and the 3D models were printed. The structures were printed using Formlabs Clear ${ }^{\mathrm{TM}}$ resin (Formlabs, USA) with the layer thickness of $50 \mu \mathrm{m}$. 3D printed fittings were postprocessed by washing with isopropanol for $10 \mathrm{~min}$ to remove uncured resin and dried in the oven at $60^{\circ} \mathrm{C}$ for $24 \mathrm{~h}$. 


\section{Fabrication of axisymmetric droplet generators}

Single emulsions. The post-processed 3D printed connector was aligned co-axially with the needle secured by $5 \mathrm{~mm}$ OD elastic tubing (Tigers Polymer Corporation, Osaka, Japan). For the outlet connection, two elastic tubes with ODs and IDs as (3 $\mathrm{mm}, 1 \mathrm{~mm}$ ) and (5 mm, $3 \mathrm{~mm}$ ) were connected. This assembly consisted of a single flow-focusing unit produced single waterin-oil (W/O) emulsions. Three different needles $(25 \mathrm{G}, 30 \mathrm{G}$, and $34 \mathrm{G}$ ) (VMATIC, Ningbo, China) with IDs of $250 \mu \mathrm{m}, 160 \mu \mathrm{m}$ and $60 \mu \mathrm{m}$ were used for the experiments to show the customizability of the device.

Double emulsions. To produce double emulsions, two assembled flow-focusing devices were connected in series with the two needles forming a co-axial arrangement. Two consecutive needles, $34 \mathrm{G}$ and $20 \mathrm{G}$ with ID as $60 \mu \mathrm{m}$ and $620 \mu \mathrm{m}$ respectively, were used. Elastic tubes with OD and ID as $(5 \mathrm{~mm}$, $3 \mathrm{~mm}$ ) were used to secure the connection between the devices, and between the device and the outlet.

Janus particles. A 3D printed Y-channel fitting specifically designed for producing co-laminar flow was assembled with the connector. Two concentric PTFE tubes (muse 2000, China) with the ODs and IDs of $(1.8 \mathrm{~mm}, 1 \mathrm{~mm})$ and $(1 \mathrm{~mm}, 0.7 \mathrm{~mm})$ were fitted to the outlet of Y-channel. The outlet connection was formed by the elastic tube with the OD and ID as ( $5 \mathrm{~mm}, 3 \mathrm{~mm})$.

\section{Droplet formation}

Single emulsions. 2\% v/v Span 80 (Sigma-Aldrich, Singapore) in hexadecane (Alfa Aesar, Scientific resources, Singapore) was used as the continuous fluid and $1 \% \mathrm{v} / \mathrm{v}$ blue dye aqueous solution was used as the dispersed phase for single emulsions. $Q_{\mathrm{c}}$ was varied between $100 \mu \mathrm{L} \mathrm{min}^{-1}$ and $1000 \mu \mathrm{L} \mathrm{min}^{-1}$ and $Q_{\mathrm{d}}$ was maintained at $10 \mu \mathrm{L} \mathrm{min}{ }^{-1}$ for droplet generation with three different needles (25 G, $30 \mathrm{G}, 34 \mathrm{G})$.

Double emulsions. Inner, middle and outer phase fluids used for the formations of $\mathrm{W} / \mathrm{O} / \mathrm{W}$ double emulsions were $0.25 \% \mathrm{v} / \mathrm{v}$ red dye aqueous solution, $3 \% \mathrm{v} / \mathrm{v}$ Span 80 in hexadecane and $0.1 \% \mathrm{v} / \mathrm{v}$ yellow dye in $90 \%$ glycerol and $10 \%$ water solution, respectively. $Q_{\mathrm{i}}$ and $Q_{\mathrm{m}}$ were maintained constant at 5

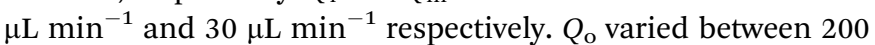
$\mu \mathrm{L} \min ^{-1}$ and $600 \mu \mathrm{L} \min ^{-1}$.

Janus particles. 1\% irgacure 1173 photoinitiator (2-hydroxy2-methylpropiophenone-97\%) (Sigma-Aldrich, Singapore) was added into poly(ethylene glycol) diacrylate (PEGDA)-700 (SigmaAldrich, Singapore) solution to form a prepolymer mixture. This prepolymer solution was colored into two different colors by adding $1 \% \mathrm{v} / \mathrm{v}$ red and green dyes. The Y-channel facilitated the flow of the two-colored solutions that merged as a single dispersed phase at the outlet. UV light source at $20 \%$ intensity was used for polymerizing the prepolymer. $Q_{\mathrm{dr}}$ was maintained at $10 \mu \mathrm{L} \mathrm{min}{ }^{-1}$ whereas three flowrates used for green stream

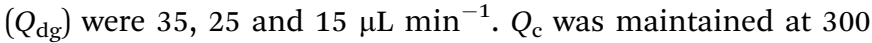

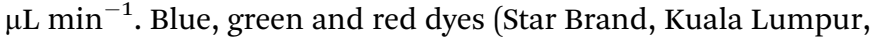
Malaysia) were used for making colored aqueous solutions. All fluids were dispensed using $10 \mathrm{~mL}$ syringes (Terumo, Tokyo, Japan) fitted to the syringe pumps (NE-4000, New Era Syringe Pump. Inc, Farmingdale, NY, USA).

\section{Imaging and characterization}

In-channel flows and collected droplets were imaged using Leica stereoscope (Leica Microsystems GmBH, Wetzlar, Germany) coupled with Basler AG camera (Basler Ace acA204090uc, Basler AG, Germany). Diameter of droplets was measured using ImageJ (ImageJ, National Institutes of Health (NIH), Bethesda, MD, USA).

\section{Conflicts of interest}

There are no conflicts to declare.

\section{Acknowledgements}

M. H. thanks for the project funding from SUTD Digital Manufacturing and Design Centre (DManD) supported by the Singapore National Research Foundation (RGDM1620503), and the infrastructure supported by SUTD-MIT International Design Centre (IDC) (IDG11700103). S. V. thanks for the President's Graduate Fellowship awarded by the Ministry of Education (MOE), Singapore..

\section{References}

1 G. M. Whitesides, Nature, 2006, 442, 368.

2 L. M. Fidalgo, G. Whyte, B. T. Ruotolo, J. L. Benesch, F. Stengel, C. Abell, C. V. Robinson and W. T. Huck, Angew. Chem., Int. Ed., 2009, 121, 3719-3722.

3 G. Wang, C. Lim, L. Chen, H. Chon, J. Choo and J. Hong, Anal. Bioanal. Chem., 2009, 394, 1827-1832.

4 I. Shestopalov, J. D. Tice and R. F. Ismagilov, Lab Chip, 2004, 4, 316-321.

5 D. Dendukuri and P. S. Doyle, Adv. Mater., 2009, 21, 40714086.

6 C.-H. Choi, J.-H. Jung, D.-W. Kim, Y.-M. Chung and C.-S. Lee, Lab Chip, 2008, 8, 1544-1551.

7 Y. H. Kim, L. Zhang, T. Yu, M. Jin, D. Qin and Y. Xia, Small, 2013, 9, 3462-3467.

8 J. H. Kim, T. Y. Jeon, T. M. Choi, T. S. Shim, S.-H. Kim and S.-M. Yang, Langmuir, 2013, 30, 1473-1488.

9 Q. Xu, M. Hashimoto, T. T. Dang, T. Hoare, D. S. Kohane, G. M. Whitesides, R. Langer and D. G. Anderson, Small, 2009, 5, 1575-1581.

10 A. Fang and B. Cathala, Colloids Surf., B, 2011, 82, 81-86.

11 J. Wan, Polymers, 2012, 4, 1084-1108.

12 L.-F. Cai, Y. Zhu, G.-S. Du and Q. Fang, Anal. Chem., 2011, 84, 446-452.

13 K. Churski, T. S. Kaminski, S. Jakiela, W. Kamysz, W. Baranska-Rybak, D. B. Weibel and P. Garstecki, Lab Chip, 2012, 12, 1629-1637.

14 D.-K. Kang, M. M. Ali, K. Zhang, S. S. Huang, E. Peterson, M. A. Digman, E. Gratton and W. Zhao, Nat. Commun., $2014,5,5427$.

15 A. C. Hatch, J. S. Fisher, A. R. Tovar, A. T. Hsieh, R. Lin, S. L. Pentoney, D. L. Yang and A. P. Lee, Lab Chip, 2011, 11, 3838-3845. 
16 Q. Wang, D. Zhang, X. Yang, H. Xu, A. Q. Shen and Y. Yang, Green Chem., 2013, 15, 2222-2229.

17 B. Ahmad, E. Stride and M. Edirisinghe, Food Bioprocess Technol., 2012, 5, 2848-2857.

18 A. A. Maan, A. Nazir, M. K. I. Khan, R. Boom and K. Schroën, J. Food Eng., 2015, 147, 1-7.

19 Y. Xia and G. M. Whitesides, Angew. Chem., Int. Ed. Engl., 1998, 37, 550-575.

20 S. Takeuchi, P. Garstecki, D. B. Weibel and G. M. Whitesides, Adv. Mater., 2005, 17, 1067-1072.

21 J. C. McDonald, D. C. Duffy, J. R. Anderson, D. T. Chiu, H. Wu, O. J. A. Schueller and G. M. Whitesides, Electrophoresis, 2000, 21, 27-40.

22 L. Donvito, L. Galluccio, A. Lombardo, G. Morabito, A. Nicolosi and M. Reno, J. Micromech. Microeng., 2015, 25, 035013.

23 T. Femmer, A. Jans, R. Eswein, N. Anwar, M. Moeller, M. Wessling and A. J. Kuehne, ACS Appl. Mater. Interfaces, 2015, 7, 12635-12638.

24 J. M. Zhang, A. A. Aguirre-Pablo, E. Q. Li, U. Buttner and S. T. Thoroddsen, RSC Adv., 2016, 6, 81120-81129.

25 J. M. Zhang, E. Q. Li, A. A. Aguirre-Pablo and S. T. Thoroddsen, $R S C$ Adv., 2016, 6, 2793-2799.

26 T. Kanai and M. Tsuchiya, Chem. Eng. J., 2016, 290, 400-404. 27 Q. Ji, J. M. Zhang, Y. Liu, X. Li, P. Lv, D. Jin and H. Duan, Sci. Rep., 2018, 8, 4791.

28 A. Utada, E. Lorenceau, D. Link, P. Kaplan, H. Stone and D. Weitz, Science, 2005, 308, 537-541.

29 E. Quevedo, J. Steinbacher and D. T. McQuade, J. Am. Chem. Soc., 2005, 127, 10498-10499.

30 M. T. Gokmen, W. Van Camp, P. J. Colver, S. A. Bon and F. E. Du Prez, Macromolecules, 2009, 42, 9289-9294.
31 S. H. Kim and D. A. Weitz, Angew. Chem., Int. Ed., 2011, 123, 8890-8893.

32 R. A. Leon, A. Z. M. Badruddoza, L. Zheng, E. W. Yeap, A. I. Toldy, K. Y. Wong, T. A. Hatton and S. A. Khan, Cryst. Growth Des., 2014, 15, 212-218.

33 P. Wu, Y. Wang, Z. Luo, Y. Li, M. Li and L. He, Lab Chip, 2014, 14, 795-798.

34 L. Zheng, M. Zhi, Y. Chan and S. A. Khan, Lab Chip, 2018, 18, 197-205.

35 J. N. Lee, C. Park and G. M. Whitesides, Anal. Chem., 2003, 75, 6544-6554.

36 J. Zhou, A. V. Ellis and N. H. Voelcker, Electrophoresis, 2010, 31, 2-16.

37 A. Rotem, A. R. Abate, A. S. Utada, V. Van Steijn and D. A. Weitz, Lab Chip, 2012, 12, 4263-4268.

38 M. Hashimoto, R. Langer and D. S. Kohane, Lab Chip, 2013, 13, 252-259.

39 Z. Zhou, T. Kong, H. Mkaouar, K. N. Salama and J. M. Zhang, Sens. Actuators, A, 2018, 280, 422-428.

40 P. Lu, L. Wu and X. Liu, Micromachines, 2017, 8, 268.

41 T. Nisisako, T. Torii, T. Takahashi and Y. Takizawa, Adv. Mater., 2006, 18, 1152-1156.

42 Z. Nie, W. Li, M. Seo, S. Xu and E. Kumacheva, J. Am. Chem. Soc., 2006, 128, 9408-9412.

43 R. K. Shah, J. W. Kim and D. A. Weitz, Adv. Mater., 2009, 21, 1949-1953.

44 K.-H. Roh, D. C. Martin and J. Lahann, Nat. Mater., 2005, 4, 759.

45 S. Yang, F. Guo, B. Kiraly, X. Mao, M. Lu, K. W. Leong and T. J. Huang, Lab Chip, 2012, 12, 2097-2102.

46 J. R. Millman, K. H. Bhatt, B. G. Prevo and O. D. Velev, Nat. Mater., 2005, 4, 98. 